\title{
The 3S Process: A Framework for Teaching AI Strategy in Business Education Navneet Bhalla
}

\author{
"I have been impressed with the urgency of doing. Knowing is \\ not enough; we must apply. Being willing is not enough; we \\ must do."
}

Leonardo da Vinci

\begin{abstract}
A gap has emerged in teaching artificial intelligence (AI) in business education, where a style of curriculum based on strategy is missing. This article presents a new framework, the 3S Process, as a method for teaching leaders how to strategically adopt AI within their organizations. At a highlevel, the 3S Process consists of three stages (Story, Strategy, and Solution), which are described in detail in the article. Stage 1: Story in the process is inspired by the Harvard Case Method to provide context for a problem. Stage 2: Strategy uses Design Thinking to produce candidate solutions. The substage of Empathy in Design Thinking plays a crucial role to reduce bias in designing AI. Virtualization technology is a tool for students to experience hands-on learning in prototype development. Stage 3: Solution is where students advocate for their conceptual AI solution in the context of the case study. $\mathrm{AI}$ is a type of complex system; therefore, students should consider feedback loops and the potential for unintended biases to enter a deployed solution. The presentation of the 3S Process in this article is conceptual. Further empirical studies, including evaluations of the $3 S$ Process in classroom settings, will be considered in the future.
\end{abstract}

\section{Introduction}

There is a growing interest in teaching artificial intelligence (AI) and machine learning (ML) in business schools around the world (S.-W., 2018). However, an acclaimed approach to teaching AI (Figure 1) in the context of business, especially in terms of entrepreneurship, remains elusive.

Based on the author's experience working with numerous corporations of varying size, current Master of Business Administration (MBA) programs that include AI can be grouped according to three styles of curricula:

1. General Technology (providing a broad overview of AI techniques),

2. Specialized Technology (in-depth instructing of AI algorithms, data science, and optimization), and,

3. Decision Making (using AI/ML to inform the decision-making process).

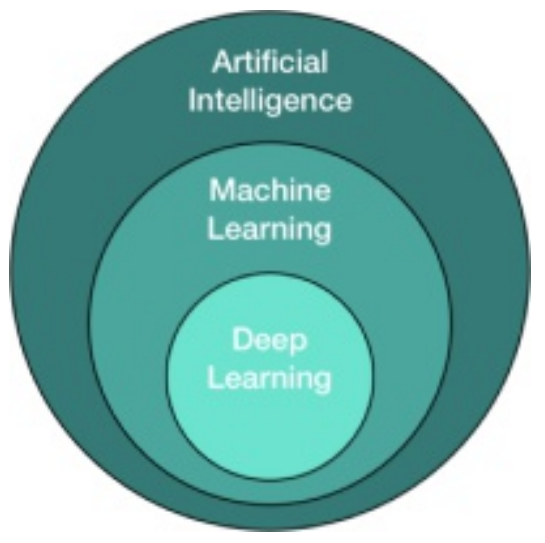

Figure 1. AI Venn Diagram. 


\section{The 3S Process: A Framework for Teaching AI Strategy in Business Education}

\section{Navneet Bhalla}

A fourth style based on strategy is missing from approaches to business education. How should leaders be educated in strategically adopting AI/ML in their organizations, and within their products and services (Stachowicz-Stanusch \& Wolfgang, 2019)? Watkins writes, "A business strategy is a set of guiding principles that, when communicated and adopted in the organization, generates a desired pattern of decision making" (2007). To glean the most from AI, it should be adopted strategically in organizations to solve business problems (and not just be another piece of technology), in order to garner exponential benefits overtime. The goal of this article is to provide a significant step towards addressing these problems by providing a new framework for a strategy-based approach, referred to as the $3 S$ Process (Bhalla, 2019).

At a high-level, the 3S Process consists of story, strategy, and solution (Figure 2). The 3S Process is inspired by the Harvard Case Method (Rebeiz, 2011) and the approach of Design Thinking (Brown, 2009). The case method provides the context for an example problem, and Design Thinking provides a strategic process for developing a considered solution. Design Thinking has been shown as an effective tool in business education, and in particular, in entrepreneurship education (Brown \& Katz, 2011).

\section{Methodology}

One of the aims of this work is to understand how to develop AI/ML in order to innovate products and services, and ultimately grow organizations. The 3S Process is the result of codifying the author's experience in teaching technical, graduate-level courses in AI and
ML (in computer science departments at universities), and the author's experience in consulting with business and technical leaders (C-suite executives) in small to medium enterprises (SMEs). It was observed that although many organizations wanted to adopt $A I$, it was not clear to them how to adopt AI. This observation fits with a survey of thousands of executives about how their companies use AI, and the data shows that only $8 \%$ of firms engage in core practices to support widespread adoption of AI (Fountaine et al., 2019). The author's objective was to devise a step-by-step process, which was based on commonly known educational techniques and strategic practices, to enable delivery of an approachable framework.

\section{Framework: The 3S Process}

Stage 1: Story is based on the Harvard Case Method. Broadly, there are four types of case situations (Ellet, 2007):

- Problems,

- Decisions,

- Evaluations, and,

- Rules.

For the purposes of the 3S Process, only case types of problems are considered (since other case types are not applicable). The intention of using a case method is to set the context of the problem to be solved. Harvard Business School (HBS) is in the midst of creating their own set of AI cases (Kenny, 2018). It will be interesting to see how HBS frames their AI cases (as well as other business schools that use case methods), and if/how the AI cases extend beyond typical problems.

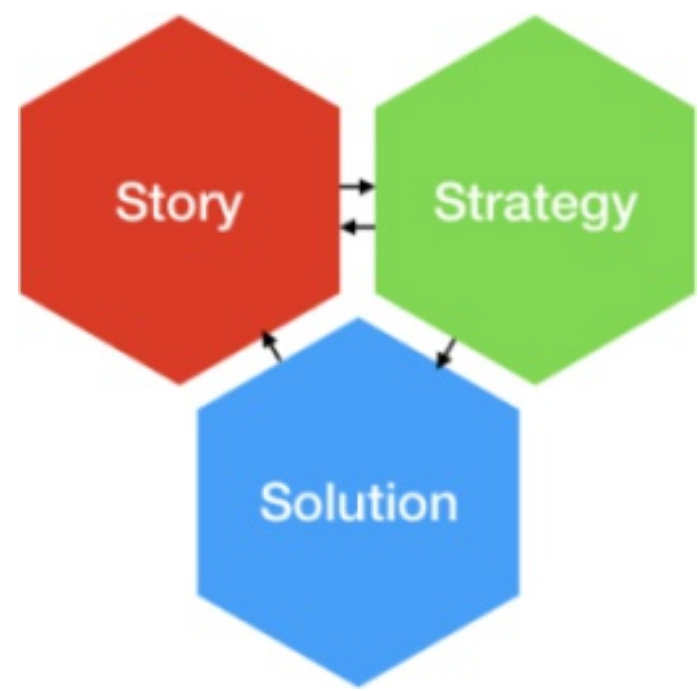

Figure 2. 3S Process 


\section{The 3S Process: A Framework for Teaching AI Strategy in Business Education}

\section{Navneet Bhalla}

Stage 2: Strategy is inspired by the approach of Design Thinking. Design Thinking was originally conceptualized for the design of physical products (Brown, 2008). Over time, Design Thinking has been applied not just to the field of industrial design, but to several others also, including the design of businesses themselves (Martin, 2009). Since its inception, there have been many variations and extensions to Design Thinking, each suited to a specific type of problem (Tschimmel, 2012). In this work, the original description of Design Thinking is used, which has five phases:

- Empathy,

- Define,

- Ideate,

- Prototype, and,

- Test.

Stage 3: Solution is the result of the Design Thinking approach within the context of a specific story. It is important to note that arriving at a solution is in actuality building an AI system (Meadows, 2008), which is integrated into another product or service. The performance, or even the behaviour itself, of the system may change with use, for example, the collection and variation of data over time.

To navigate through the framework, the 3S Process is subdivided into nine substages (Figure 3 ). The graph, with substages as nodes and with transitions from one substage to another as directed edges, represents common paths through the 3S Process. The connectivity (traversals through the graph) should be adapted to the problem to be solved. Table 1 provides a high-level description for each of the nine substages.

\section{Stage 1: Story - Scenario}

A case study, provided by educators to students, establishes the context of the problem space. Equally as important, the case study is the basis for discussion between students and educators.

\section{Stage 1: Story - Research}

Conduct research to better understand the problem space. What are the important details regarding the problem? What aspects of the problem space can be ignored? Narrow the scope of the problem, focus.

\section{Stage 2: Strategy - Empathy}

Understand the potential biases, for example, training data, particular algorithms, and potential users. Examine the problem from multiple opposing viewpoints (Martin, 2009). What are the privacy and security concerns?

\section{Stage 2: Strategy - Define}

What exactly is the problem to be solved? Define a set of quantitative/qualitative metrics to measure the success of a solution for solving the problem.

\section{Stage 2: Strategy - Ideate}

Brainstorm several candidate solutions. What are the

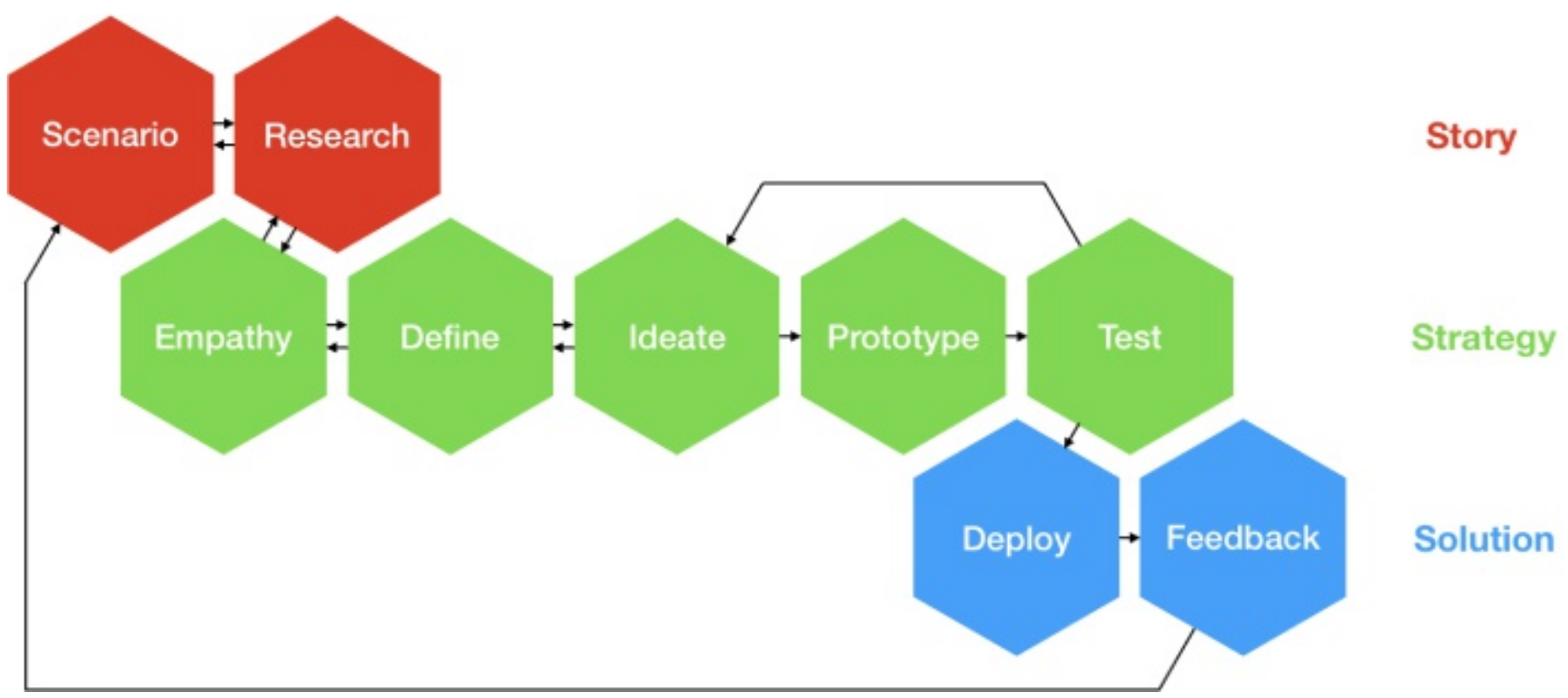

Figure 3. Graph of the nine substages of the 3S Process. 


\section{The 3S Process: A Framework for Teaching AI Strategy in Business Education Navneet Bhalla}

Table 1. Description of the nine substages of the 3S Process

\begin{tabular}{|c|c|c|}
\hline Stage & Substage & Description \\
\hline \multirow[t]{2}{*}{ Story } & Scenario & $\begin{array}{l}\text { Understand the context of the general } \\
\text { problem space. }\end{array}$ \\
\hline & Research & $\begin{array}{l}\text { Uncover the details and nuances of the } \\
\text { problem space. }\end{array}$ \\
\hline \multirow[t]{5}{*}{ Strategy } & Empathy & Anticipate and properly address biases. \\
\hline & Define & Articulate the specific problem to be solved. \\
\hline & Ideate & $\begin{array}{l}\text { Brainstorm approaches to solve the } \\
\text { problem. }\end{array}$ \\
\hline & Prototype & Design candidate solutions. \\
\hline & Test & Run trials and measure the results. \\
\hline \multirow[t]{2}{*}{ Solution } & Deploy & Release the selected solution. \\
\hline & Feedback & $\begin{array}{l}\text { Process the information returning to the } \\
\text { system. }\end{array}$ \\
\hline
\end{tabular}

available resources (for example, data and infrastructure)? If a full, candidate solution cannot be implemented as a prototype in a classroom setting, can a subset of the problem be addressed?

\section{Stage 2: Strategy - Prototype}

Ideally, a prototype should be designed quickly and implemented efficiently. Fast prototyping leads to the possibility for a greater number of iterations of the Ideate-Prototype-Test cycle.

\section{Stage 2: Strategy - Test}

Perform quantitative and qualitative measurements to evaluate the level of success of the candidate solution. If possible, compare the candidate solution to other solutions that were tested previously, and compare to other solutions in the market (or discussed in the case study).

\section{Stage 3: Solution - Deploy}

In the context of the case study, make persuasive arguments for the reasoning behind the selected solution. How would the adoption of the selected solution be marketed externally of the organization, or sold internally within the organization? How would the performance of the selected AI system be monitored over time?
Stage 3: Solution - Feedback

How will the transition from training data to continuous data be managed? What derived data can be realized?

\section{Important Features of the Framework}

There are three important features of this framework. First, the step of Empathy in Design Thinking is used to help address ethical issues when developing and deploying an AI solution. Second, a software stack using virtualization technology is discussed for how AI prototypes can be developed in practice. Third, complex systems are examined, since even a simple set of rules and algorithms can lead to unpredictable results. Complexity is an important, but often ignored aspect of AI, which is ultimately the pursuit of designing a complex system that displays agency.

\section{Empathy}

One of the greatest aspects to Design Thinking is in the phase Empathy. The ability for a designer to empathize with the end customer (and other stakeholders in the design-production-consumption process) for a product in the context of its environment leads to more humancentric and sustainable solutions. In the 3S Process, the designer is to be empathetic to reduce bias in the end solutions, be it for human-to-machine or machine-tomachine interfaces. 


\section{The 3S Process: A Framework for Teaching AI Strategy in Business Education Navneet Bhalla}

For example, Microsoft Inc. released Tay, a chat-bot, in March 2016 (Johnston, 2017). Tay used Twitter as the interface to converse with humans. By people posting offensive Tweets to Tay, the chat-bot quickly learned and then started to post its own inflammatory Tweets. Tay was taken down after only 16 hours of public operation. By employing the stage of Empathy to this research project, the developers could have anticipated the possibility of such an outcome and could have added measures to their AI chat-bot to mitigate bias.

\section{Virtualization}

While Stage 1: Story, with the case method at its core, is purely an intellectual exercise, Stage 2: Strategy offers the opportunity for learning through practical examples and exercises with software. It would be difficult, if not impossible due to time constraints, for students (for example, in an MBA course on AI) to implement a fullfledged AI system in the context of solving a case study problem. Instead, the emphasis should be on implementing a solution that addresses a subproblem, as a way to gain experience in AI through hands-on learning.

Virtualization software, for example, Docker (Boettiger, 2015), can be used as part of the Ideate-Prototype-Test substages of the 3S Process. Docker performs operatingsystem-level virtualization and runs software packages referred to as containers. Containers are isolated from each other and bundle their own application, tools, libraries, and configuration files. Containers can communicate with each other using specific channels and message passing. Docker works with operating systems that run on desktop personal computers and servers. Therefore, the focus here can be thought of as AI running in the cloud and not at the edge (that is, embedded $\mathrm{AI}$ ).

The idea here is that educators develop software that is built on top of virtualization technology (Figure 4), thus allowing students to focus on the code, algorithms, and concepts needed to build prototypes to address specific subproblems. Depending on the technical know-how of the students, they could work at a high-level (that is, determining effects based on adjusting parameters), at a low-level (write the code for specific algorithms), or somewhere in between these two positions.

There are five advantages to using virtualization software from an educational perspective.

1. Cross-platform. This allows the software to be available to a wider audience, and independent of the host operating systems (macOS, Windows, and many distributions of the Linux operating system).

2. Software bundles. The particular software needed can be used and pre-configured (for example, prepopulating a database).

3. Customizable. Specific applications can be written that run on top of Docker (for example, Python programs, which can use the vast number of $\mathrm{AI} / \mathrm{ML}$ packages that are readily available).

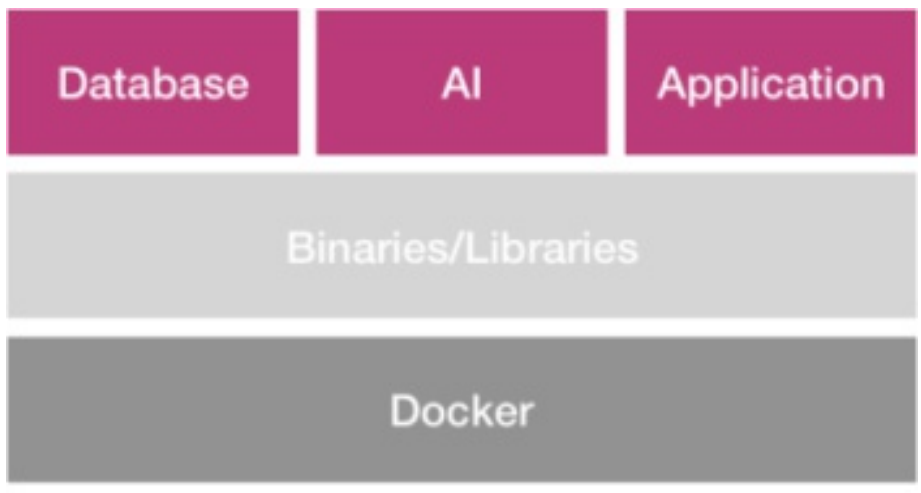

\section{Host Operating System}

Server

Figure 4. Docker stack. 


\section{The 3S Process: A Framework for Teaching AI Strategy in Business Education Navneet Bhalla}

\section{Modular. Each software bundle running on top of Docker can be developed and updated independently, meaning that educators can take a step-by-step approach to creating curriculum.}

5. Cloud-ready. Containers can be integrated into web services for production (that is, use the code that was developed for a prototype as part of the code base for the solution).

Furthermore, developing web services offer the opportunity to integrate with other cloud services (for example, Amazon Web Services, Microsoft Azure, Google Cloud, IBM Watson), through application programming interfaces (APIs), resulting in faster prototyping, access to pre-trained AI models, and continuously receiving new capabilities. Interoperability between web services based on virtual containers is one of the best methods to realize powerful, complex AI systems today.

\section{Complexity}

Stage 1: Story is based on the Harvard Case Method to provide context to a problem space, and Stage 2: Strategy uses Design Thinking and virtualization to develop practical prototypes to address subproblems. Stage 3: Solution completes the 3S Process. Since it is not reasonable for students to implement a productionready AI system in a classroom setting, the best practice would be for students to develop persuasive arguments for their particular, conceptual solutions, and try to anticipate unintended consequences. Unexpected behaviour can occur in AI due to it being a type of complex system.

Mitchell defined a "complex system" this way: "A system in which large networks of components with no central control and simple rules of operation give rise to complex collective behaviour, sophisticated information processing, and adaptation via learning or evolution." (2009)

Information returning to an AI system can be considered as either as a positive feedback loop (amplification) or a negative feedback loop (dampening). It is critical to understand the information returning to the system, the correct method to process the information, and the best practice to store the information. For example, unexpected feedback changed the behaviour of the chat-bot Tay, as discussed previously.

\section{Conclusion}

To summarize, this article presents a new framework, the 3S Process, for teaching AI in the context of business education. Stage 1: Story uses the Harvard case method to set the context of the problem space. Students are expected to engage in discussion to further understand the problem at hand, to uncover details and narrow the scope of the problem space. Stage 2: Strategy is based on the approach of Design Thinking to develop a prototype, which for practical purposes in a classroom setting addresses a subproblem unveiled in the case study. Particular emphasis is placed on the substage of Empathy to reduce potential biases in the final AI system. Furthermore, virtualization software can be used to create practical candidate solutions, and thus provide a hands-on learning opportunity for the IdeatePrototype-Test substage cycle. Stage 3: Solution is where students advocate for their conceptual AI solution in the context of the case study and describe their Design Thinking thought process to reach their AI solution. Students should remember that AI is a type of complex system and postulate potential feedback loops, while taking into account the potential for unintended biases to enter the system.

When educators use the 3S Process the expectation should not be that business students develop a deep, technical understanding of AI. Instead, the hope is that the 3S Process provides students with critical thinking and hands-on experience with AI, so that they can make more informed strategic decisions about $\mathrm{AI}$ as leaders in their future organization and as part of teams. Business education using the 3S Process can equip leaders with common language and understating regarding AI, thereby improving communication between management and technical experts.

It should be noted that the 3S Process can be adapted from use in education to be applied to entrepreneurship. Instead of using a case study, Stage 1: Story is based on the business problem to be solved and context is provided by market realities. Instead of addressing a subproblem, Stage 2: Strategy directly addresses the business problem. As with the education case, leaders should be aware of bias in the business case as well. The use of virtualization software at this stage has a real benefit, as it can be transferred with ease to production, Stage 3: Solution, particularly for cloud services. Leaders will have to sell their solutions 


\section{The 3S Process: A Framework for Teaching AI Strategy in Business Education}

\section{Navneet Bhalla}

internally within their organization and measure external market response. Complexity will still play a factor and require leaders to continually monitor the performance of their AI system.

Finally, the 3S Process is a complex network itself. The author's intent is that leaders can leverage the 3S Process, and that the resulting collective behaviour will lead to the emergence of creative thinking around integrating $\mathrm{AI}$ in business.

\section{Acknowledgements}

Many thanks to the two anonymous reviewers \& TIM Review editors for their valuable feedback.

\section{References}

Bhalla, N. 3S Process: Re-Envisioning AI in Business Education. ISPIM Connects Ottawa, 2019. 1-9.

Boettiger, C. 2015. An introduction to Docker for reproducible research. ACM SIGOPS Operating Systems Review - Special Issue on Repeatability and Sharing of Experimental Artifacts: 71-79.

Brown, T. 2008. Design Thinking. Harvard Business Review.

Brown, T. 2009. Change by Design: How Design Thinking Transforms Organizations and Inspires Innovation. New York City, NY, HarperBusiness.

Brown, T. \& Katz, B. 2011. Change by Design. Product Innovation Management, 29: 381-383.

Ellet, W. 2007. The Case Study Handbook: How to Read, Discuss, and Write Persuasively About Cases. Boston, MA, Harvard Business School Press.

Johnston, I. 2017. AI Robots Learning Racism, Sexism, and Other Prejudices from Humans, Study Finds. The Independent. Available:

https://www.independent.co.uk/life-style/gadgetsand-tech/news/ai-robots-artificial-intelligenceracism-sexism-prejudice-bias-language-learn-fromhumans-a7683161.html [Accessed February 25 2019].

Kenny, B. 2018. Harvard Business School to Expand Case Studies Related to Artificial Intelligence. Harvard Business School. Available: https://www.hbs.edu/news/releases/Pages/stephenschwarzman-gift-artificial-intelligence.aspx [Accessed February 5 2019].

Martin, R. 2009. The Design of Business: Why Design Thinking is the Next Competitive Advantage. Boston, MA, Harvard Business Review Press.

Martin, R. 2009. The Opposable Mind: Winning Through Integrative Thinking. Boston, MA, Harvard Business School Publishing.

Meadows, D. H. 2008. Thinking in Systems: A Primer.
White River Junction, VT, Chelsea Green Publishing.

Mitchell, M. 2009. Complexity: A Guided Tour. Oxford, Oxford University Press.

Rebeiz, K. S. 2011. An Insider Perspective on Implementing the Harvard Case Study Method in Business Teaching. US-China Education Review, A, 5: 591-601.

Stachowicz-Stanusch, A. \& Wolfgang, A. (Eds.) 2019. Management and Business Education in the Time of Artificial Intelligence: A Need to Rethink, Retrain, and Redesign. Charlotte, Information Age Publishing.

S.-W., C. 2018. Rise of the Machines. The Economist. Available:

https://www.economist.com/whichmba/rise-

machines [Accessed February 5 2019].

Tschimmel, K. 2012. Design Thinking as an effective Toolkit for Innovation. XXIII ISPIM Conference: Action for Innovation: Innovating from Experience, 2012 Barcelona.

Watkins, M.D. 2007. Demistifying strategy: The What, Who, How, and Why. Harvard Business Review.

\section{About the Author}

Navneet Bhalla, $\mathrm{PhD}$, is a Senior Honorary Research Associate at University College London, in the Department of Computer Science, and a member of Intelligent Systems Group. He is also the founder of Cetana AI Inc., a consultancy specializing in artificial intelligence. Prior to starting the consultancy, Navneet was a postdoctoral fellow at Harvard University (in the Department of Chemistry and Chemical Biology), at Cornell University (in the Department of Mechanical and Aerospace Engineering), and at the Universität Paderborn (in the Department of Computer Science). His research interests include self-assembling systems, machine learning, soft robotics, mechanical design, composite materials, and innovation management.

Citation: Bhalla, N. 2019. The 3S Process: A Framework for
Teaching AI Strategy in Business Education. Technology Innovation Management Review, 9(12): 36-42.

http://doi.org/10.22215/timreview/1290 (cc) BY

Keywords: 3S Process, Artificial Intelligence, Business Education, Design Thinking, Harvard Case Method 